\title{
PESQUISA E FORMAÇÃO ESPECIALIZADA EM CONSERVAÇÃO DE MATERIAIS PÉTREOS: DA ARQUITETURA À INDÚSTRIA LÍTICA; DA ARTE ACADÊMICA AOS OBJETOS DE ARTE MODERNA E CONTEMPORÂNEA
}

\author{
Yacy-Ara Froner ${ }^{1}$, Luiz Antônio Cruz Souza ${ }^{2}$ \\ 1- Professora UFMG, Coordenadora do LAPA-MHNJB-UFMG; 2 - Professor UFMG, Coordenador do LACICOR-CECOR-EBA-UFMG \\ froner@ufmg.br
}

\begin{abstract}
Resumo: Serra da Capivara (PI), Sambaquis Catarinenses (SC), a indústria lítica da tradição Umbu do sul do país (RS), muiraquitãs amazonas $(A M)$, Congonhas e Inhotim (MG). A investigação no campo de conservação de materiais pétreos pode não ser a primeira coisa que vem à mente ao anunciar esses locais, e isso ocorre porque a pesquisa em Arquitetura, Arqueologia ou no campo da História da Arte nem sempre foca questões de materialidade e de preservação nos estudos acerca da cultura material. Este trabalho pretende fornecer uma visão geral da investigação sobre a conservação do patrimônio construído em pedra a partir de uma revisão da literatura. Do período entre guerras ao contexto atual, autores como Price \& Doehne (2010), entre outros, têm alargado o debate em torno do tema e gerado um campo sinergético de estudos. Quais foram as mudanças substanciais ocorridas nas últimas décadas? Além de uma formação especializada decorrente do International Stone Conservation Course promovido pelo ICCROM desde 1976, inúmeros laboratórios, centros de pesquisas e programas de pós-graduação têm investido em estudos transdisciplinares como parte do pensamento complexo da ciência pós-moderna. Este pensamento abarca distintos atores dos mais diversos campos de pesquisa com o intuito de promover uma visão ampliada da área.
\end{abstract}

Palavras Chave: formação em conservação, acervos líticos, materiais pétreos

\begin{abstract}
RESEARCH AND SPECIALIZED EDUCATION IN CONSERVATION OF STONE MATERIALS: FROM ARCHITECTURE TO LYTIC INDUSTRY; FROM ACADEMIC ART TO MODERN AND CONTEMPORARY ART OBJECTS. Serra da Capivara (PI-Brazil), Sambaquis Catarinenses (SC-Brazil), lithical industry of South (RS), muiraquitãs from Amazonas (AM), Congonhas and Inhotim (MG-Brazil). Research in the conservation of stone materials field is not the first thing that we think when we named these places, and it happens because the research in the field of architecture, archaeology and art history do not always focuses on materiality issues and conservation studies about of material culture. This work aims to provide an overview of research on the conservation of the heritage built in stone from a literature review. Authors such as Price \& Doehne (2010), among others, have extended the debate on the issue and generated a synergetic field studies. What were the major changes that have occurred in recent decades in the field of stone conservation? In addition to specialized training due to the International Stone Conservation Course organized by ICCROM since 1976, numerous laboratories, research centers and graduate programs have endowed in transdisciplinary studies as part of the complex thinking of postmodern science. This thought includes various actors from several fields of research in order to promote an enlarged view of the area.
\end{abstract}

Keywords: conservation training, lithic collections, stone material

\section{INTRODUÇÃO}

O reconhecimento científico, acadêmico e profissional da área de Conservação-Restauração de Bens Culturais - assim como as implicações políticas e sociais no que tange à preservação do Patrimônio Cultural em distintos contextos - pode ser observada em uma conjuntura internacional de longa duração.

No séc. XIX, as primeiras faculdades visaram à formação de uma elite burguesa e em distintos países - incluindo Brasil e Portugal - as universidades demarcaram territorialidades. 0 bacharel, geralmente circunscrito a um grupo restrito, assumia um papel fundamental no contexto social, uma vez que a formação investida de um capital simbólico do saber, determinava a hierarquia desses indivíduos em sistemas específicos da política, da cultura e da ciência. Como em inúmeras áreas de conhecimento, a conformação do campo do Patrimônio Cultural gerenciou, nesse sistema, a subordinação de uma expertise vinculada a outros campos de saber.

Herdeira das profundas alterações no projeto

doi: 10.18285/geonomos.v24i2.852 educacional europeu - como a criação das École Polytechnique (1794), École Normale (1794) e do Conservatoire des Arts et Métiers (1794) - a partir da Revolução Francesa e das reformas napoleônicas, a formação nos campos das da Arquitetura, da Arqueologia, da Conservação, da Museologia, da História da Arte e da Arte compartilhou o território de gênese das Belas Artes; da mesma forma que distintas disciplinas encontraram seus caminhos preliminares nos estudos politécnicos e nas ciências dos materiais, principalmente no que compete a função utilitária do saber na Revolução Industrial e no sistema capitalista. Nesse contexto, moldado pela Revolução Industrial, despontam os primeiros teóricos da conservação no campo da arquitetura: John Ruskin (1819-1900), com "The Seven Lamps of Architecture", de 1849; Eugène Emmanuel Viollet-leDuc (1814-1879), com “Dictionnaire Raisonné de I'Architecture Française du XI au XVI Siècle", de 1868 e Camillo Boito (1836-1914), promotor do " $3^{\circ}$ Congresso degli Ingegneri e Architetti italiani" de Roma em 1883, o qual gerou a "Carta Italiana del Restauro".

Derivam dessas bases os principais teóricos do início do século XX, como Gustavo Giovannoni 
(1873-1947) e Luca Beltrami (1854-1933). Coube a Le-Duc o desenvolvimento de uma metodologia de restauração dos monumentos franceses góticos em pedra, cujos parâmetros são seguidos ainda hoje, como a intervenção a partir do reconhecimento do estilo e a utilização de materiais idênticos ou similares aos originais associados ao uso de materiais contemporâneos, como as estruturas de ferro utilizadas na época. A Escola de Viena também determinou uma sistematização do pensamento, uma vez que seus principais teóricos combinaram uma carreira acadêmica com atividades curatoriais em museus. A obra "Der moderne Denkmalkultus" de 1903 de Alois Riegl (1858-1905) foi forjada sob a demanda de organização da Comissão de Monumentos Históricos da Áustria e, seguindo a mesma linha de pensamento, Max Dvořák (18741921) publicou "Catecismo da Preservação de Monumentos" em 1916 (FRONER, 2016a).

Contudo, o surgimento de uma Ciência moderna voltada ao estudo e à preservação do patrimônio, sistematizada por práticas institucionais como publicações científicas, eventos, estruturação de laboratórios e discussões sobre formação, apenas ocorreu após a Primeira Guerra Mundial, com a inauguração do Escritório Internacional de Museus em 1926.

1.1. As conferências de Roma, Atenas e Cairo nos anos trinta e a mudança dos paradigmas da preservação

Os anos seguintes à I Grande Guerra (1914-1919) promoveram profundas alterações nos modelos de formação científica e nas noções ampliadas da cultura e da preservação do patrimônio. Pela primeira vez Educação, Ciência, Patrimônio e Cultura são vistos de forma ampliada por meio de um esforço internacional. Questões cruciais caras à área ainda hoje - formação; ciência aplicada aos estudos dos materiais constitutivos das obras, dos fatores de degradação e dos métodos de intervenção; cooperação e divulgação de pesquisas - foram amplamente discutidas, alterando profundamente as relações entre a Ciência, a Conservação, a Restauração e a Preservação de Bens Culturais.

Três eventos transformaram o modelo da prática amadora da intervenção: a "Conferência Internacional para o Estudo dos Métodos Científicos Aplicados ao Exame e Conservação de Obras de Arte", realizada em Roma em 1930, a "Conferência Internacional para o Estudo dos problemas relativos à proteção e conservação de Monumentos de Arte e História", realizado em Atenas em 1931, e a "Conferência Internacional para o Estudo dos Problemas Administrativos e Técnicos concernentes às Escavações", realizado no Cairo em 1937.
Dentre as recomendações comuns resultantes desses encontros estão: o uso de exames científicos antes da limpeza e da restauração, bem como a documentação fotográfica e gráfica dos registros do processo de intervenção; a validação científica dos métodos e materiais de intervenção e a demanda de uma formação especializada. Os temas apresentados nessas conferências estão diretamente ligados aos trabalhos de ciência e inovação desenvolvidos nas pesquisas dos laboratórios estruturados após a Primeira Guerra Mundial. Aproximadamente vinte e cinco laboratórios estabelecidos em universidades, superintendências patrimoniais e museus da Europa, América do Norte e Ásia podem ser mapeados na documentação gerada por estes eventos. Estas Conferências foram decisivas para lidar de forma subsidiada com as questões de difusão, conservação, restauração e pesquisa de bens culturais. A partir de então, quatro questões primordiais foram colocadas ao métier: formação, reconhecimento profissional, proteção legal e compartilhamento de pesquisa.

No âmbito de estudos de preservação de materiais pétreos, inúmeras pesquisas acerca de metodologias científicas de análise, conservação e restauração de bens culturais artísticos, arqueológicos e arquitetônicos foram apresentadas nesses encontros, demonstrando a pertinência desse campo interdisciplinar de estudo.

\section{A FORMAÇÃO ESPECIALIZADA E OS LABORATÓRIOS DE PESQUISA NA PRIMEIRA METADE DO SÉCULO XX}

Laboratórios de ciências aplicadas ao estudo, conservação e restauração de bens culturais surgiram simultaneamente ao desenvolvimento de uma formação especializada. Em 1936, o Escritório Internacional de Museus publicou o texto "L'examen scientifique des œuvres d'art et leur restauration", reforçando o papel da organização na divulgação do conhecimento, visando o intercâmbio de pesquisas e pesquisadores. O texto assinala o papel do Prof. Renato Mancia (1885-?) de Milão na direção de um laboratório técnico destinado à formação de conservadores e restauradores de museus. No suplemento de abril, aparece a indicação da publicação de seu livro, "L'esame scientifico delle opera d'arte ed il loro restauro" (1936).

Os dois volumes comentados nessa resenha objetivam demarcar o caráter inédito da integração entre o laboratório científico e a formação de conservadores, já referenciado por Alda Levi (18901950) no seu texto "L'analyse chimique des terres cuites anciennes en vue de l'identification de leur provenance", por meio de sua atuação no Instituto Politécnico da Universidade de Milão. 
Sous la forme d'un appendice au premier volume, - mais qui peut server de conclusion logique à l'ouvrage, - l'auteur publie le programme de l'École nationale de Restauration qu'il a inaugurée à Milan, le 14 décembre 1935 . Ce programme divise les matières en trois sections; matières techniques, matières artistiques et matières scientifiques. Dans le discours qu'il a prononcé à l'occasion de cette inauguration, M. Mancia, après avoir esquissé la série des examens scientifiques, a insisté sur l'apport considérable que des recherches de cet ordre constituent non seulement pour la sauvegarde rationnelle du patrimoine artistique, mais aussi dans l'ordre des connaissances œuvres d'art et particulièrement dans le domaine de l'archéologie (OIM, 1936, p.161).

O Escritório entende que contextos distintos determinarão formas distintas de organização na formação.

La formation professionnelle des restaurateurs, que a été mise à l'étude également dans le revue Mouseion, est certainement le but auquel doivent tender les autorités préposées à la conservation de œuvres d'art. L'institutions fondée en Italie et les créations similaires que nous avons signalées en leur temps en d'autres pays, ainsi que les laboratoires attachés aux grands musées d'Europe et d'Amérique, témoignent de la compréhension qui si manifeste à l'heure actuelle pour le sort de œuvres du passé, menacées et parfois compromises par des interventions malhabiles plus encore que par l'action du temps. (OIM, 1936, p.161)

Esta compreensão atualizada sobre as transformações no sistema museal e na prática das intervenções em monumentos e obras de arte irá também discutir o atrito desnecessário entre Ciência e História da Arte no campo dos estudos e da preservação. Reforça o fato de que, após o impacto do encontro de Roma, a compreensão da demanda de uma interação recíproca e complementar entre os campos de saber apenas contribui de forma sinergética na construção do conhecimento estruturado pela área. Nesse sentido, não apenas o métier deveria ser contemplado com o conhecimento proporcionado pela visibilidade dos estudos, mas o público também deveria se beneficiar desta doutrina forjada pela compreensão da materialidade e da conceituação de uma obra (arquitetural, arqueológica e/ou artística).

\subsection{As primeiras pesquisas e publicações em torno da conservação de acervos pétreos}

Ao longo dos anos trinta, as discussões geradas não conseguiram um consenso em relação à formação e regulamentação da profissão. No entanto, os resultados imediatos desta movimentação em torno do Estado da Arte da Preservação foram significativos: o aporte e a projeção internacional da Carta de Atenas, uma das primeiras recomendações internacionais; os documentos "Manuel de la conservation des peintures" (elaborado entre 1933-1939), "La conservation des monuments d'art et d'histoire" (dois volumes publicados em 1933), "Museographie: architecture et aménagement des musées d'art", de 1935 e "Manuel de la technique des fouilles archéologiques", de 1939, dirigidos aos gestores de museus, sítios e monumentos. Além disso, diversos documentos produzidos no período demarcam a discussão dos aspectos legais relacionados à proteção de bens culturais no âmbito internacional, os quais devem ser atribuídos ao Escritório e contemplados à luz das transformações do paradigma contemporâneo da preservação. No contexto norte-americano, diversos laboratórios integrados aos museus e universidades também alteraram o modelo de atuação:

Around that time, Museum laboratories were starting to sprout up in major institutions on the East Coast: in 1929, the Boston Museum of Fine Arts founded its Scientific Research Laboratory and in 1930, the Metropolitan Museum in New York founded its Chemical Laboratory. Science was finding its place in the museum world, and as deterioration mechanisms became better understood, the interest in preventive conservation grew (LAMBERT, 2014, p.11).

Vários pesquisadores da época atuaram especificamente no campo da conservação de materiais pétreos. Nas atas das palestras proferidas na conferência de Roma, é possível mapear alguns nomes de pesquisadores, bem suas instituições de origem. A maioria dessas publicações foram publicadas na revista "Mouseion" editada pelo Escritório Internacional de Museus.

Representando a Superintendência do Patrimônio da Iugoslávia, Georges Bochkovitch (1899-?), arquiteto e conservador do Museu de Arte e de História de Belgrado, apresentou a comunicação "La restauration des sculptures de l'église du Monastère de Kalénic", publicada na “Mouseion" em 1932 (Figuras 1 e 2).

No texto, Bochkovitch descreve o processo de recuperação

Une fois la reconstruction décidée, il s'agissait de se borner à préserver les parties originales, sans rien entreprendre qui pût changer l'aspect original de l'édifice. Cependant, quand on enleva la couche de mortier, on se trouva en présence d'une partie importante, reconstruite en 1766. On avait procédé à ces travaux en utilisant comme matériel de construction tout ce qui était antérieurement tombé de l'église. En ce faisant, on avait tourné vers l'intérieur du mur le côté travaillé de ces divers fragments - profils de corniches ou ornements d'archivoltes -, n'en laissant voir 
désormais que le verso, qu'on se contenta d'égaliser et de façonner de manière très sommaire.

A palestra de James Rorimer (1905-1966), "L'application des Rayons ultraviolets à l'examen des sculptures en marbres", divulgou as primeiras experiências em arqueometria desenvolvidas nos EUA. Formado em química pela Universidade de Harvard em 1927, nesse mesmo ano começou a trabalhar como assistente no Departamento de Artes Decorativas da instituição e dois anos depois como curador do Departamento de Arte Medieval do Metropolitan Museum of Art (EUA). Previamente, em 1929, Rorimer havia publicado no volume 8 da "Mouseion" os avanços preliminares do laboratório, mas sua apresentação do dia 13 de outubro de 1930 na Conferência de Roma aparece apenas nas atas do evento, não sendo publicada pelo Escritório Internacional de Museus. No ano seguinte ao encontro, este conteúdo fará parte de seu livro "Ultra-Violet Rays and their use in the examination of Works of Art".

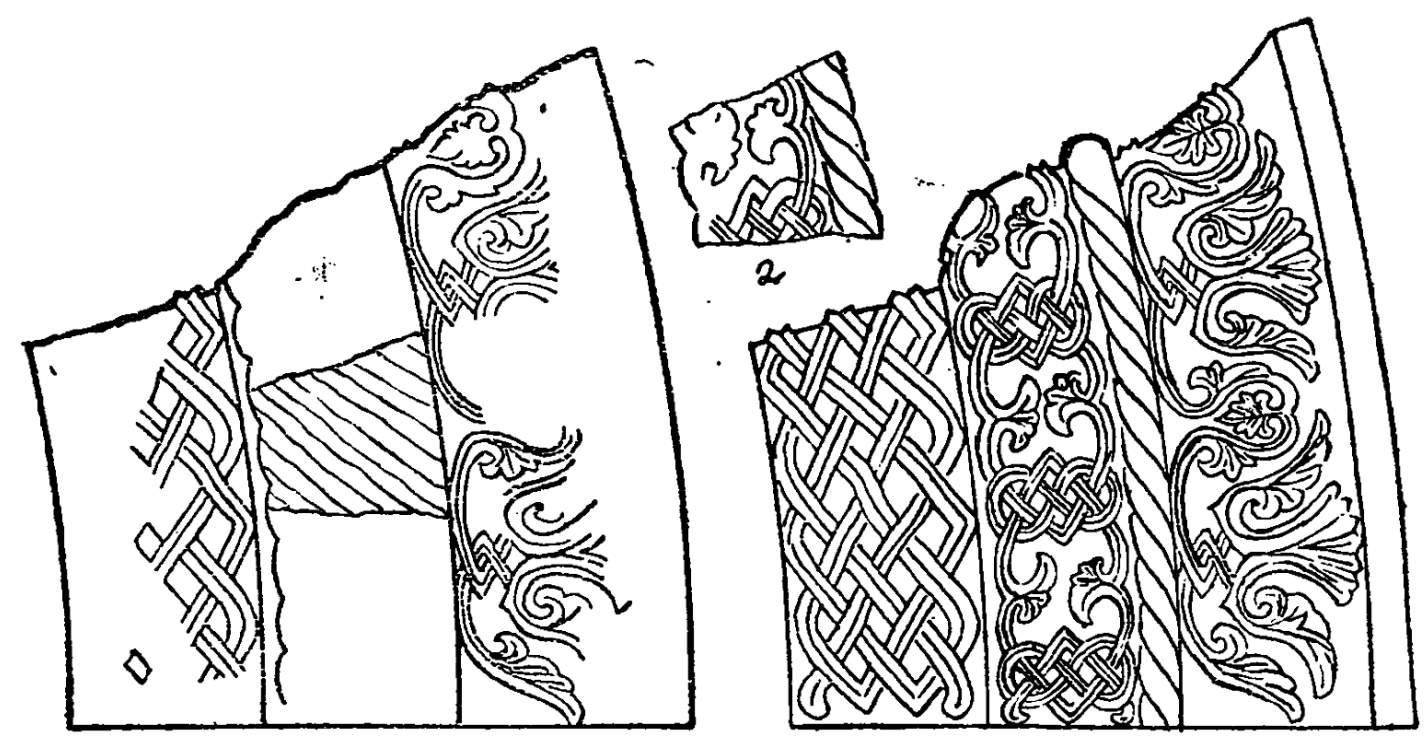

Figura 1. Recuperação da banda da arquivolta completamente destruída. Crédito: "Mouseion", v.17-18, 1932, gallica.bnf.fr / Bibliothèque de I'INHA / coll. J. Doucet

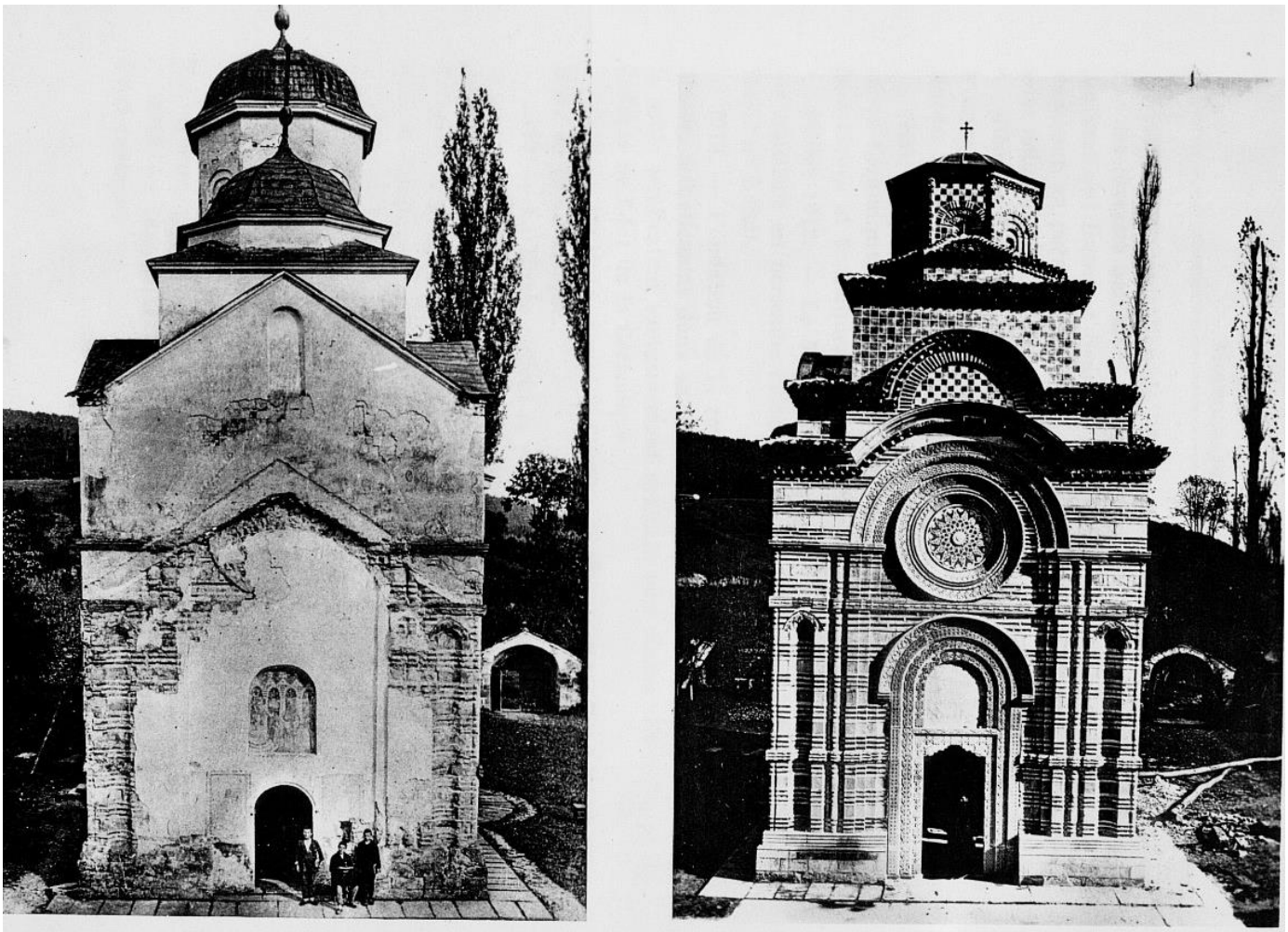

Figura 2. Recuperação da Igreja de Kalic. Crédito: "Mouseion", v.17-18, 1932, gallica.bnf.fr / Bibliothèque de I'INHA / coll. J. Doucet 
Benoit Champion (1863-1952), arqueólogo e chefe do laboratório do Musée des Antiquités Nationals de St. Germain, em Lyon, apresentou a conferência "De la nécessité d'une technique d'observation sous forme d'analyses descriptives, pour l'identification des objectes archéologiques", publicada posteriormente com o título "Identification et conservation des objets préhistoriques" (Figura 3 - a, b), na edição da revista em 1931.

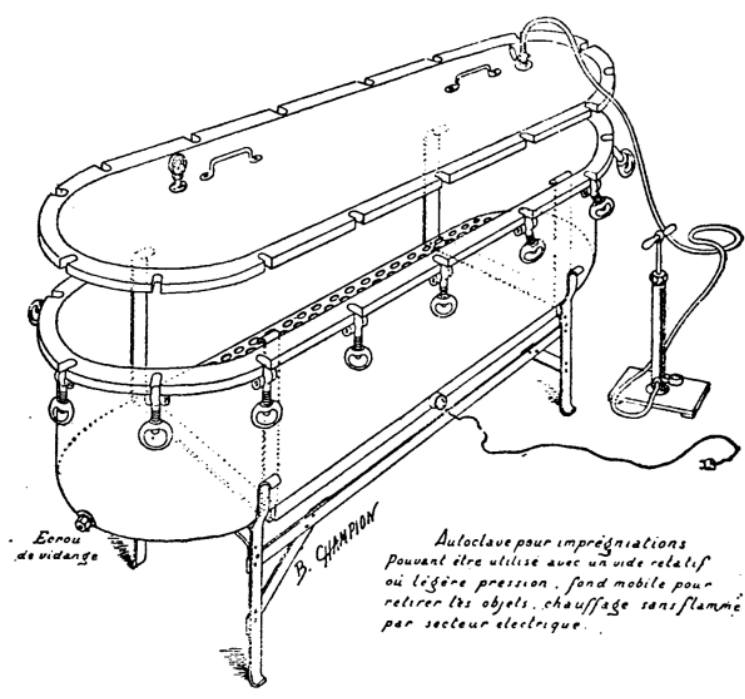

Figura 3. Esquema de autoclave de impregnação. Crédito: "Mouseion", v.16, 1931, gallica.bnf.fr / Bibliothèque de I'INHA / coll. J. Doucet

Jean Fernand Cellerier (1870-1936), diretor do Laboratoire d'Recherches Scientifiques do Museu do Louvre e do Laboratoire d'essais du Conservatoire des Arts et Métiers de Paris, apresentou o trabalho “Mesures à prendre pour préserver les œuvres d'art en pierre contre les atteintes de l'atmosphère", publicado em 1932. Anteriormente ele já havia publicado no volume $7 \mathrm{da}$ "Mouseion" estudos relativos aos exames científicos de pinturas murais de Berzé la Ville, o qual também foi apresentado no encontro de Roma. Em seu texto, ele sistematiza os métodos de ensaio para análise de materiais pétreos (Figura 4).

Da Alemanha, Carl Brittner (1883-1958), diretor do Laboratório de Química do Museu do Estado de Berlim expôs sua pesquisa "Procédée de nettoyage des sculptures en pierre", também publicado em 1933.

Da Itália, Gino Fogolari (1875-1941) Superintendente de Monumentos de Veneza, apresentou a conferência "Anciens procédés employés à Venise pour la préservation des œuvres $d^{\prime}$ 'art en pierre contre les atteintes de l'atmosphère para l'application d'un enduit et possibilité d'utiliser aujourd'hui ces procèdes". Contudo, seu trabalho não chegou a ser pulicado, sendo posteriormente citado no volume 23-24 de 1933.

\begin{tabular}{|c|c|}
\hline ESSAIS & OBSERVATIONS \\
\hline Examen pétrographique. & $\begin{array}{l}\text { Etat minéralogique des grains consti- } \\
\text { tuants, degre d'agglomération des } \\
\text { grains, nature et compression et struc- } \\
\text { ture du cément, stratification, etc. }\end{array}$ \\
\hline $\begin{array}{l}\text { Délermination de la résistance du } \\
\text { cément. }\end{array}$ & Procédé Hirschwald. \\
\hline Essai de ramollissement de l'eau. & $\begin{array}{l}\text { Essai de résistance à la traction et à la la } \\
\text { compression, à la dureté, soit à l'état } \\
\text { sec, soit après séjour dans l'eau. }\end{array}$ \\
\hline Essai de résistance à la gelée. & $\begin{array}{l}\text { Mode opératoire du Laboratoire d'Es- } \\
\text { sais, antérieurement décrit par M. LE- } \\
\text { DUc, ancien Chef de Section. }\end{array}$ \\
\hline $\begin{array}{l}\text { Détermination de la porosité abso- } \\
\text { lae et de la porosité relative et } \\
\text { de l'absorption d'eau. }\end{array}$ & Procédé mécanique. \\
\hline Imprégnation par teinte. & \\
\hline $\begin{array}{l}\text { Analyse chimique des constituants } \\
\text { particuliers. }\end{array}$ & $\begin{array}{l}\text { Complétant l'examen pétrographique } \\
\left(\mathrm{FeS}^{2} \text {, carbone, } \mathrm{CaO}, \mathrm{MgO}^{2}, \mathrm{FeO}, \mathrm{Al}^{2} \mathrm{O}^{3}\right) \text {. }\end{array}$ \\
\hline
\end{tabular}

Figura 4. Tabela sistemática de ensaios de materiais pétreos. Crédito: "Mouseion", v.19, 1932, gallica.bnf.fr / Bibliothèque de I'INHA / coll. J. Doucet

A partir de um cruzamento entre os campos da Arquitetura, da Arqueologia e da História da Arte, diversos trabalhos foram apresentados no campo de estudos científicos e métodos de preservação de pinturas murais, incluindo intervenções das estruturas construtivas em pedra. Francesco Valenti (1869-1953), Superintendente de Monumentos da Sicília, apresentou e publicou o trabalho gerenciado pelo instituto, sob o título "Travaux exécutés en vue de la conservation de mosaïques du Dôme de Messine et leur transport sur un nouveau support", publicado em 1932; Roberto Papini (1883-1957) apresentou o trabalho "Physiologie et pathologie des peintures murales"; George Leslie Stout (18971978), "Transport des fresques orientales sur de nouveaux supports", publicado com Rutherford J. Gettens em 1932; Demetrio Helbing, Membro do Comitê Nacional de Química da Itália e do Escritório Nacional de Museus da Bélgica, apresentou a conferência "La conservation de fresques". No programa do evento, consta uma comunicação proferida pelo Secretariado do Escritório Internacional de Museus intitulada "Protection des peintures murales contre les moisissures", determinando a importância do assunto para o Escritório. No entanto, no levantamento da documentação existente no Fondo Pellati não há o "Proces-verbal" da seção, impossibilitando a identificação do comunicador e do conteúdo apresentado.

As conferências seguintes, em Atenas (1931) e Cairo (1937), produziram uma vasta literatura sobre métodos de análise e preservação de materiais pétreos em projetos relacionados à restauração arquitetônica e estudos arqueológicos, determinando a interdisciplinaridade desta tipologia de pesquisa. 


\section{O CURSO INTERNACIONAL DE CONSERVAÇÃO DE PEDRAS PROMOVIDO PELO ICCROM}

Após a Segunda Guerra Mundial (1939-1945), novamente uma ampla movimentação internacional em torno das restaurações, reconstruções e repatriações do pós-guerra são gerenciadas por organismos internacionais de cooperação. Como bem observa Pierre Leveau, as fundações da primeira metade do século $\mathrm{XX}$ forjaram as instituições atuais.

En 1926, l'Office international des Musées (OIM) fut par exemple créé pour fédérer les institutions muséales sur le plan international, avant que le Conseil international des Musées (ICOM) le fasse en 1946. De même, une Commission internationale des Monuments historiques (CIMH) avait été instituée en 1934 pour construire le réseau que le Conseil international des Monuments et des Sites (ICOMOS) développe depuis sa création en 1965. Un Comité international des Experts archivistes (CIEA) avait également été créé en 1930 pour s'acquitter de la mission qui est devenue celle du Comité international des Archives (ICA) en 1948. Tous ces organismes dépendaient à l'époque de l'Organisation de Coopération intellectuelle $(\mathrm{OCl})$, qui était à la Société des Nations (SDN) ce que I'UNESCO est à I'ONU. On pourrait développer encore cette analogie structurelle qui s'explique par la nécessité de restaurer, en 1919 puis en 1945 , le réseau des sociétés savantes coupé par les guerres (LEVEAU, 2014, p.2).

$\mathrm{Na}$ esteira destas observações, convém pontuar que a criação de um "Comitê de Experts em Estudos Científicos de Bens Culturais" apenas ocorreu de fato em 1956 a partir da constituição do ICCROM International Centre for the Study of the Preservation and Restoration of Cultural Property.

Entre 1966 e 1976, o ICCROM irá formatar os primeiros cursos de formação na área, com áreas de concentração em torno da Conservação em Arquitetura (1966), Conservação de Pintura Mural (1968), Ciência da Conservação (1973), Conservação Preventiva (1975) e Conservação de Pedras (1976).

Over the last decades, Conservation-restoration, Conservation Science and particularly Preventive Conservation have developed their methodological and epistemological knowledge on ideas coming from other areas of knowledge. More than any other field, these branches of science have been fed with discoveries and procedures from other areas while elaborating the theories and methods that are only applicable to its particular milieu. Architecture and Archaeological Conservation, Conservation Science, and Preventive Conservation are the priority areas that can be identified in the early historical courses offered by ICCROM between the 1960s and 1970s. They not only educated people but they also brought together the most influential researchers in the area, and through their programmes, they organized and systematized new theories that contributed to the epistemological expansion of this field of study (FRONER, 2016b, p.13).

Laboratórios especializados, intercâmbio de cientistas e a elaboração de um currículo sólido voltado para profissionais atuantes e já qualificados ao nível da graduação. Inúmeros pesquisadores, incluindo Jukka Jokilehto e Nicholas Stanley-Price, foram alunos e posteriormente professores dos cursos de formação do ICCROM.

O "Curso de Conservação de Pedras", criado em 1976, ofereceu dezenove edições até 2015, sendo que esta última ocorreu em Roma, em parceria com o Getty Conservation Institute (GCI).

Desde o início, o curso adotou uma abordagem colaborativa e multidisciplinar, projetada para atender profissionais envolvidos na conservação de estruturas de pedra de monumentos históricos e arqueológicos, além de artefatos e objetos artísticos. O principal objetivo do curso é, por meio da teoria e da prática, subsidiar o exercício da conservação da pedra a partir de princípios científicos. Um currículo multidisciplinar, incluindo trabalho de campo, proporciona aos participantes uma compreensão holística dos fatores de degradação e dos processos de deterioração de estruturas e objetos em pedra. Ao disseminar metodologias, técnicas e materiais validados pela Ciência da Conservação, o curso promove uma compreensão prática dos métodos de conservação-restauração apropriados e estratégias de gestão de longo prazo. Através de palestras, debates, sessões de laboratório, demonstrações, visitas e atividades práticas, os participantes discutem tanto as teorias fundamentais da área, como avaliam os avanços na tecnológicos e de pesquisa que têm influenciado as abordagens práticas no que diz respeito a todas as fases de conservação da pedra.

Exercícios de campo em grupo visam a experiência da prática coletiva do diagnóstico, do projeto e da intervenção, oferecendo a oportunidade de lidar com cenários reais de trabalho os quais demandam soluções multidisciplinares e colaboração. Durante o curso, a formação prévia de alunos e professores é compartilhada, reconhecendo, assim, que a experiência coletiva considera o sinergismo entre distintas especializações na busca de soluções mais eficazes no campo da conservação em materiais pétreos.

O curso é dividido em seis unidades principais distribuídas onze semanas. Estas unidades incluem tópicos específicos, tais como: princípios e teorias da Conservação; ciências dos materiais como uma ferramenta para a identificação, análise e concepção 
de tratamentos de conservação; mecanismos de deterioração; técnicas de diagnóstico para a identificação de causas e efeitos dos fatores de degradação; metodologia de avaliação do estado de conservação de acervos; estratégias de conservação para ações imediatas e de longo prazo, incluindo a prevenção, manutenção, reparação e tratamento; técnicas práticas de conservação, gestão de projeto de conservação de pedra, e o valor do trabalho dentro de equipes multidisciplinares (ICCROM, 2015).

Eric Doehne e Clifford A. Price, no trabalho intitulado "Stone Conservation: An Overview of Current Research" (2010), apontam os principais pesquisadores e avanços tecnológicos e metodológicos da área nas últimas décadas, muitos deles produzidos por professores e ex-alunos dos cursos formativos do ICCROM. Os autores citam uma série de pesquisas envolvendo novas tecnologias, sendo um dos principais livros de referência no campo da investigação e preservação da cultura material em pedra.

Atualmente, a amplitude de pesquisas na área pode ser encontrada na potência dos estudos analíticos sobre marcas de ferramenta, tecnologia de construção, caracterização e desempenho através da Arqueometria e da Ciência da Conservação; métodos de documentação a partir do uso de escaneamento a laser 3D, calibração de imagens, sistemas informatizados de bases de dados, critérios de acesso e programas interativos; estudos no campo da Conservação Preventiva visando estabilização do microclima, mitigação de flutuações ambientais, avaliação de estruturas com base em modelos computacionais; estudos de métodos e desempenho de materiais de intervenções com ampla aplicação, incluindo uso de nanotecnologia, laser e produtos industriais; estudos sócio-ecônomicos, ambientais, históricos e museais relacionados ao uso e função social do patrimônio material pétreo, incluindo projetos inclusivos e de interação com o público externo e com a comunidade local, a promoção circuitos culturais, museus de território. Desse modo, a introdução de novas tecnologias, métodos de estudo, intervenção e uso tem, atualmente, modificado o campo da preservação da cultura material em pedra.

\section{CONSIDERAÇÕES FINAIS}

A gama de investigações no que tange a preservação da cultura material em pedra pode ser observada em uma variedade de bens culturais: da arquitetura à indústria lítica, da arte acadêmica aos objetos de arte moderna e contemporânea, o patrimônio em pedra brasileiro manifesta sua riqueza em inúmeros exemplos, como as pinturas rupestres da Serra da Capivara (PI), os petroglifos catarinenses (SC), a indústria lítica da tradição Umbu do sul do país (RS), os muiraquitãs amazônicos (AM), os profetas de Congonhas ou as obras em mármore de Waldemar Cordeiro em Inhotim (MG) (Figura 5 $a, b, c, d, e, f)$.

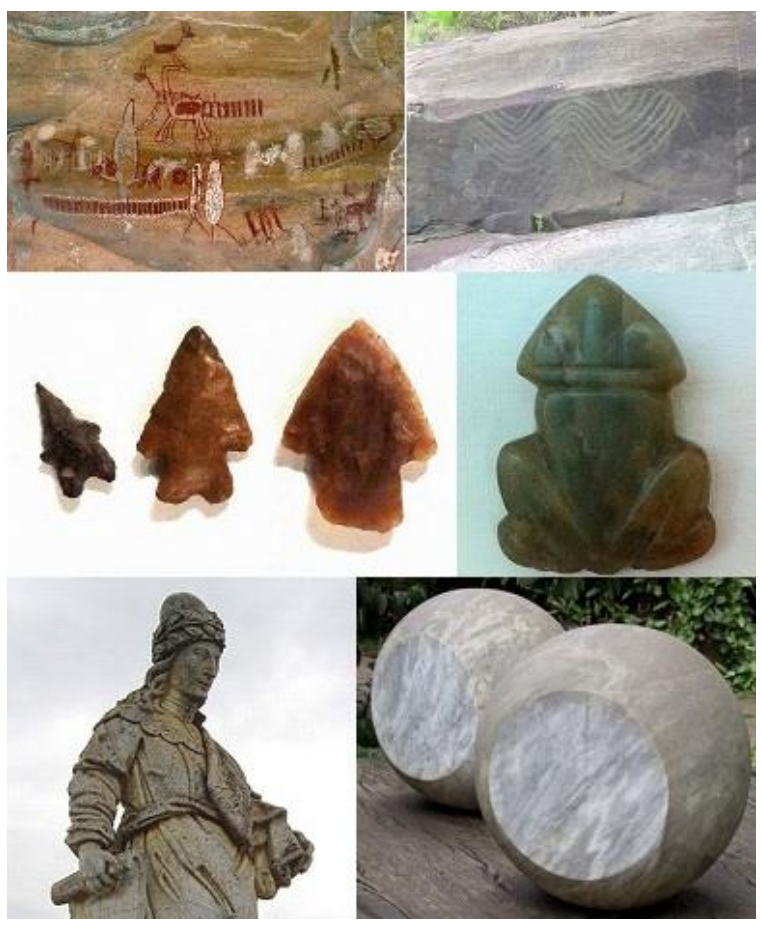

Figura 5. (a, b, c, d, e, f)-Arte em pedra brasileira

Para além das questões específicas relacionadas à cultura material de acervos pétreos, a preservação das jazidas em torno dos assentamentos urbanos se estabelece como um fator primordial para o estabelecimento de conexões potenciais no campo da História da Arte Técnica, bem como em relação à Ciência da Conservação. O reconhecimento das áreas de extração pode elucidar questões, tais como o trânsito de matéria-prima, a qualidade do material empregado e sua vulnerabilidade intrínseca.

Desse modo, a noção de proteção do patrimônio geológico não apenas impacta questões relacionadas à Paisagem Cultural, mas princípios fundamentais pertinentes à própria história do crescimento urbano, principalmente das primeiras povoações brasileiras, vinculadas à arquitetura e à arte local.

Apenas uma formação especializada e multidisciplinar de agentes que lidam com este patrimônio - arquitetos, engenheiros, cientistas da conservação, historiadores, arqueólogos, geólogos, conservadores - pode subsidiar um trabalho acurado de pesquisa e preservação.

O curso de conservação de pedras do ICCROM inaugurou, historicamente, um dos primeiros cursos na área. Contudo, a demanda de formação de investigadores, laboratórios de pesquisa e produção de literatura especializada, cada vez mais determina 
que processos de formação ocorram em espaços acadêmicos. O modelo de seu programa e seu formato pode ser replicado e adaptado em contextos específicos, principalmente em projetos colaborativos entre países de língua portuguesa e universidades.

Redes colaborativas voltadas ao estudo, caracterização, análise de procedência, uso e preservação de pedras ornamentais empregadas na arquitetura, em bens integrados e bens culturais móveis são extremamente importantes. Eventos, como este simpósio de Caracterização e Conservação da Pedra - emblematicamente realizado no Museu de Congonhas - possibilita a construção de pesquisas compartilhadas por meio de encontro de profissionais, pesquisadores e cientistas da área.

\section{REFERÊNCIAS BIBLIOGRÁFICAS}

DOEHNE, E.; PRICE, C.A. Stone conservation: an overview of current research. Santa Monica [CA-EUA]: Getty Conservation Institute, 2010.

OIM. Mouseion. Paris: Office international des Musées, Société des nations, 1927-1946.

FRONER, Y.A. Demandas históricas: a constituição da Ciência da Conservação e a formação do Conservador-Restaurador. Conservar Património. Lisboa, v.23, junho 2016a, p. 15-23,
URL: http://revista.arp.org.pt/pdf/2015048.pdf, DOI: 10.14568/cp2015048.

FRONER, Y.A. Intellectual Co-operation Institutions for Cultural Heritage Protection: History, Science, Training, and Politics. Roma: ICCROM/CAPES, 2016b (Tese pós-doutoral).

LAMBERT, Simon. The Early History of Preventive Conservation in Great Britain and the United States (1850-1950). CeROArt, Paris, n.9, janvier 2014. UR: http://ceroart.revues.org/3765. Acesso: Junho 2016.

LEVEAU, Pierre. L'enquête sur la formation des restaurateurs dans I'Entre-Deux-Guerres: transformation d'un métier et reconnaissance d'une profession (1929-2011). CeROArt, Paris, n.9, janvier 2014, URL: http://ceroart.revues.org/3772. Acesso: Junho 2016.

ICCROM. http://www.iccrom.org/it/nineteenth-internationalcourse-on-stone-conservation/. Acesso: Junho 2016.

Contribuição ao

1ํ. Simpósio Brasileiro de Caracterização e Conservação da Pedra 14 a 16 de dezembro de 2016, Congonhas - MG

Nota:

É de responsabilidade da comissão editorial do Simpósio a revisão gramatical, ortográfica, de citações e referências bibliográficas. As normas de submissão podem se diferenciar das desta revista. 